\title{
Intraoperative Monitoring of Hypoglossal Nerve Using Hypoglossal Motor Evoked Potential in Infratentorial Tumor Surgery: A Report of Two Cases
}

\author{
Seung Yeun Kim, MD ${ }^{1}$, Hyo Won Im, MD ${ }^{1}$, Young-Doo Choi, BS $^{2}$, Keewon Kim, MD, PhD ${ }^{1}$, \\ Jin Wook Kim, MD, PhD², Yong Hwy Kim, MD, $\mathrm{PhD}^{2}$, Han Gil Seo, $\mathrm{MD}^{1}$
}

Departments of ${ }^{1}$ Rehabilitation Medicine and ${ }^{2}$ Neurosurgery, Seoul National University Hospital, Seoul, Korea

The hypoglossal nerve (CN XII) may be placed at risk during posterior fossa surgeries. The use of intraoperative monitoring (IOM), including the utilization of spontaneous and triggered electromyography (EMG), from tongue muscles innervated by CN XII has been used to reduce these risks. However, there were few reports regarding the intraoperative transcranial motor evoked potential (MEP) of hypoglossal nerve from the tongue muscles. For this reason, we report here two cases of intraoperative hypoglossal MEP monitoring in brain surgery as an indicator of hypoglossal deficits. Although the amplitude of the MEP was reduced in both patients, only in the case 1 whose MEP was disappeared demonstrated the neurological deficits of the hypoglossal nerve. Therefore, the disappearance of the hypoglossal MEP recorded from the tongue, could be considered a predictor of the postoperative hypoglossal nerve deficits.

Keywords Intraoperative monitoring, Motor evoked potentials, Hypoglossal nerve

\section{INTRODUCTION}

Resecting tumors in an infratentorial lesion requires a high surgical precision, because in that operable area many lower cranial nerves (LCN; CN IX-XII) nuclei exist [1-3]. In that case, the LCN play an important role in swallowing and speech function and deficits of these nerves lead to dysphagia, dysarthria, and hoarseness. Therefore, preserving the function of LCN during infratentorial surgery is one of the most important goals to neurosurgeons performing these types of surgical procedures. Additionally, the intraoperative monitoring (IOM)

Received July 14, 2017; Accepted September 4, 2017

Corresponding author: Han Gil Seo

Department of Rehabilitation Medicine, Seoul National University Hospital, 101 Daehak-ro, Jongno-gu, Seoul 03080, Korea. Tel: +82-2-2072-1659, Fax: +82-2-743-7473, E-mail: hangil_seo@snuh.org; hgseo80@gmail.com

ORCID: Seung Yeun Kim (http://orcid.org/0000-0002-0994-8055); Hyo Won Im (http://orcid.org/0000-0002-5254-1728); Young-Doo Choi (http:// orcid.org/0000-0002-1674-1095); Keewon Kim (http://orcid.org/0000-0001-6597-578X); Jin Wook Kim (http://orcid.org/0000-0002-3773-3940); Yong Hwy Kim (http://orcid.org/0000-0001-9009-4191); Han Gil Seo (http://orcid.org/0000-0001-6904-7542).

(c) This is an open-access article distributed under the terms of the Creative Commons Attribution Non-Commercial License (http://creativecommons.org/ licenses/by-nc/4.0) which permits unrestricted noncommercial use, distribution, and reproduction in any medium, provided the original work is properly cited. Copyright @ 2018 by Korean Academy of Rehabilitation Medicine 
can help localize nerves and prevent nerve injury during surgery [1-3].

For the IOM of LCN, in the same as other cranial nerves, a spontaneous electromyography (s-EMG), triggered electromyography (t-EMG) and transcranial motor evoked potential (TcMEP) could be used. Among them, the TcMEP is the only the indirect real-time technique to monitor the nerve function without approaching to the nerves. And there are many reports about monitoring CN IX, CN $\mathrm{X}$, and CN XI by TcMEP as seen in a literature review [110]. However, the techniques to monitor the function of CN XII are restricted in s-EMG and t-EMG, and the use of TcMEP of CN XII has been neglected until now.

Here, we report two cases of IOM of the CN XII using hypoglossal TcMEP with s-EMG in infratentorial surgery, and the result of a postoperative swallowing function. These cases offer the potential result that the IOM of hypoglossal TcMEP could be used as an indicator for detecting injury of the hypoglossal nerve and predict postoperative complications.

\section{CASE REPORTS}

\section{Case 1}

A 33-year-old female with a 2-year history of dizziness was presented for review. The patient had no past medical history. The patient noted that her incidence of dizziness was getting worse, and a newly-onset bilateral nystagmus on the lateral gaze was presented on March 15,2016 . Therefore, it was recommended that the patient undergo a brain magnetic resonance imaging (MRI) on
April 4, 2016. The brain MRI showed an approximate 3.4 $\mathrm{cm} \times 3.6 \mathrm{~cm} \times 5.7 \mathrm{~cm}$ sized high signal intensity (HSI), nonenhancing mass on the T2-weighted images at fourth ventricle (Fig. 1A), and the formal reading of the MRI finding was a suspicious epidermoid cyst. The preoperative physical examinations showed dizziness and nystagmus on the bilateral lateral gaze. To rule out the LCN deficits, the neurosurgeons tested the loss of taste at the posterior one-third of the tongue (for $\mathrm{CN} \mathrm{XI),} \mathrm{gag} \mathrm{reflex}$ (for CN XI, CN X), the presence of uvula deviation (for CN $\mathrm{X}$ ), weakness of sternomastoid and/or trapezius muscle (for CN XI), and the presence of tongue deviation, dysarthria (for CN XII). The result of testing found that there was no abnormal finding on these tests. Then, on May 23, 2016, the patient was admitted at the Department of Neurosurgery and underwent a tumor removal surgery on May 24, 2016.

\section{Case 2}

A 38-year-old female suffered from a 1-month history of diplopia and dizziness. The patient had no history of any other diseases. After visiting a local clinic, the patient was recommended to undergo a brain MRI. Next, she took a brain MRI on April 28, 2016. The brain MRI showed an approximately measured $5.2 \mathrm{~cm} \times 5.4 \mathrm{~cm} \times 4.8 \mathrm{~cm}$ HSI mass at the fourth ventricle on T2-weighted images, with strong diffusion restriction and scalloped expansion into bilateral cerebellum and brain stem (Fig. 1B). As the previous case, the patient was diagnosed with a suspicious epidermoid cyst at the fourth ventricle. On the preoperative physical examinations, the incidence of nystagmus
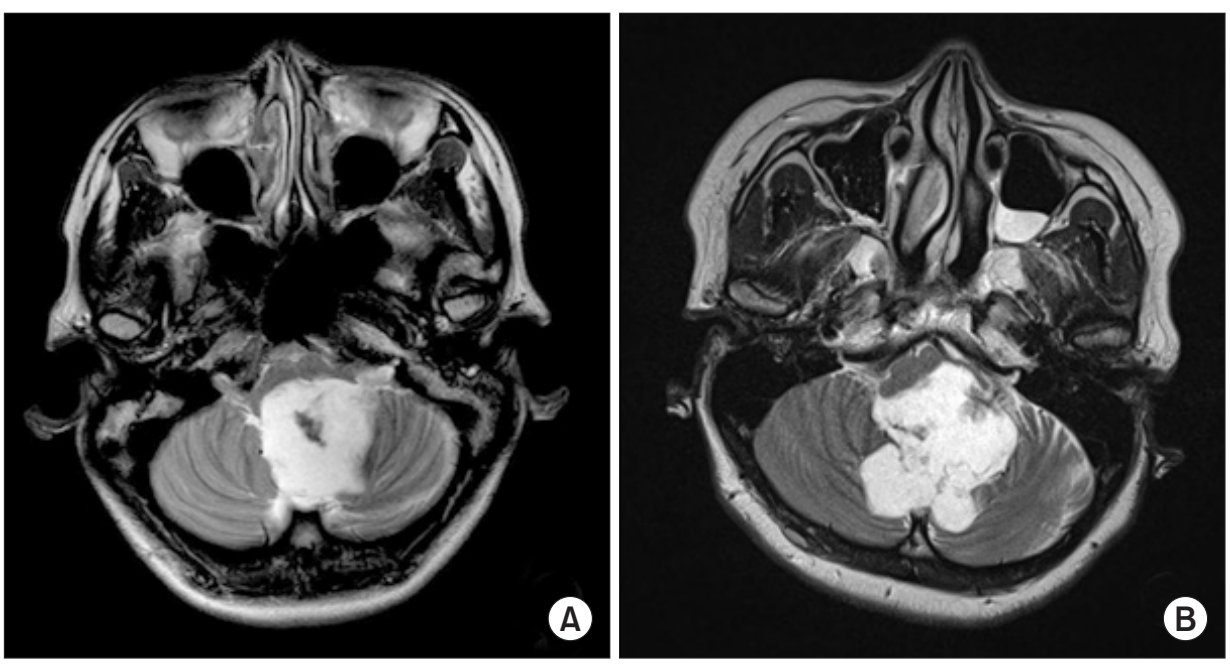

Fig. 1. The preoperative T2-weighted images of brain magnetic resonance imaging showed $3.4 \mathrm{~cm} \times 3.6$ $\mathrm{cm} \times 5.7 \mathrm{~cm}$ mass in case $1(\mathrm{~A})$ and $5.2 \mathrm{~cm} \times 5.4 \mathrm{~cm} \times 4.8 \mathrm{~cm}$ mass in case 2 (B) at the fourth ventricle. 
was seen in all directions and the left facial sense was decreased at the V2 area. Except for those noted and reviewed findings, there was no abnormality on the review of the lower cranial exams which were same tests in case 1. Therefore, the patient was admitted at the Department of Neurosurgery and went through tumor removal surgery on May 24, 2016.

\section{Surgical procedure and technique of motor evoked potential from tongue}

In both of the patients, the TcMEP and s-EMG were intra-operatively recorded from the tongue during surgery. For recording the signal from the tongue muscles, the needle electrodes were placed across the midline of the oral tongue with bipolar configuration (Fig. 2). After insertion of the needle, the tongue was put into the mouth, and the lip was sealed to prevent bite injury of the tongue. Then, the wire of recording electrode was fixed with tape to maintain the placement of the needle. For the completion of the transcranial electrical stimulation, the needle electrodes were inserted on the scalp at C3 and C4 according to the International 10-20 System for electrode placement. Thereafter, there were trains of five pulses (biphasic square) stimulus were applied by a commercialized transcortical stimulator (Eclipse; Axon Systems Inc., Hauppauge, NY, USA). The frequency of the trains was $1 \mathrm{~Hz}$, and an inter-stimuli interval (ISI)

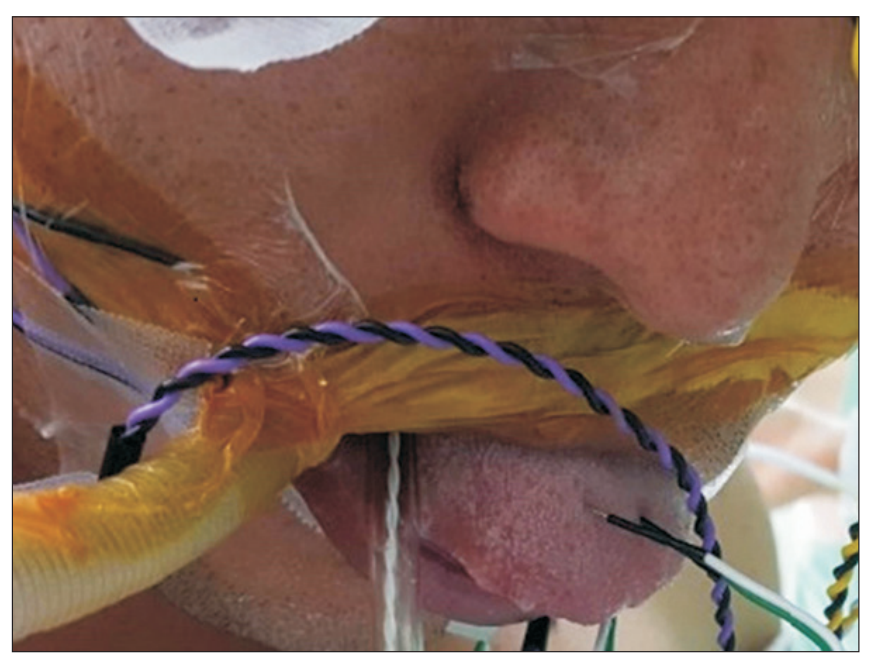

Fig. 2. The recording needle electrodes for motor evoked potential and spontaneous electromyography of the hypoglossal nerve were inserted on the midline of the oral tongue. was 1-2 ms. The average intensity of stimulus was 350 V. However, when an amplitude of MEP was recorded as below $200 \mu \mathrm{V}$, the stimulation intensity was gradually increased up to $450 \mathrm{~V}$ until stable MEP amplitude above $200 \mu$ was obtained in all target muscles. The signals were filtered between 30 and 1,000 Hz. Beyond the IOM of the hypoglossal nerve, and we also performed an additional IOM including the TcMEP and the s-EMG at the bilateral trapezius, thenar, and abductor hallucis muscles and only the s-EMG at the bilateral orbicularis oculi and oris muscles. The somatosensory evoked potential (SSEP) at bilateral median and posterior tibial nerves and brainstem auditory evoked responses (BAER) were also monitored. The anesthesia was induced and maintained using continuous infusion of propofol $(20 \mathrm{mg} / \mathrm{mL} \times 50 \mathrm{~mL}=1,000$ $\mathrm{mg})$ and remifentanil $(20 \mathrm{mcg} / \mathrm{mL} \times 50 \mathrm{~mL}=1 \mathrm{mg})$. During that timeframe, the rocuronium ( $50 \mathrm{mg}$ ) was injected intravenously before the tracheal intubation to help the procedure of intubation and ventilation. During the surgical procedure, the physiatrist played a role as technological supervisor and real time interpreter. If there were abnormal waveforms and/or decreased signals, the physiatrist gave immediate feedback to the surgeons and helped to establish a real time plan to alter a surgical plan if the situation presented itself as being necessary at that time.

\section{Results of hypoglossal MEP and clinical course \\ Case 1}

It was noted that a whitish, keratinous mass was exposed between cerebellar tonsils and the tumor dissection procedure was then initiated on the patient. During the tumor dissection around the facial colliculus and hypoglossal trigone, the tonic discharges from the s-EMG of the tongue and right orbicularis muscles were first monitored and repeated (Fig. 3B). Then, at 20 minutes later, when the tumor at hypoglossal and vagal trigone was removed, the amplitude of the MEP from the tongue was not detected and did not recover until the end of surgery (Fig. 3A). After the surgery, the nystagmus on the bilateral lateral gaze and the dizziness were noted as improved. However, the patient complained of newly onset dysphagia after the surgery. On physical examinations, there were tongue deviation to the right side and tongue fasciculation. When the patient was asked to poke the inside of her cheek by tongue, the patient was unable to complete 


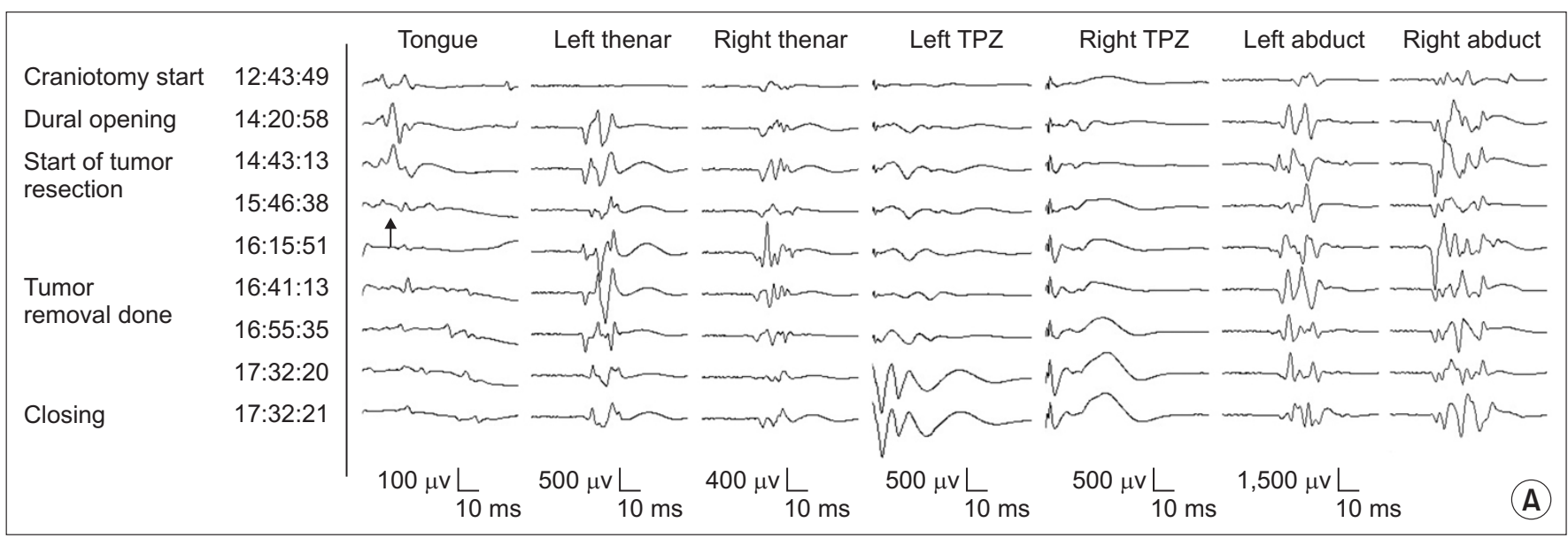

$15: 29$

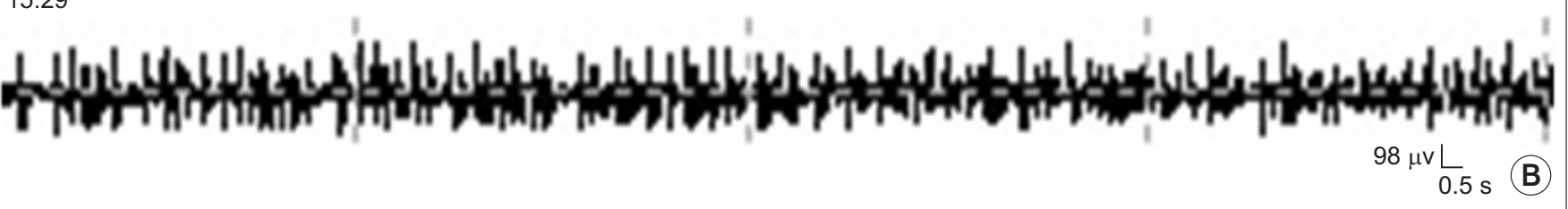

Fig. 3. The tongue and other muscles MEP (A) and spontaneous tongue EMG (B) recorded in case 1. During surgery, the tonic discharge from the spontaneous EMG of tongue muscles was monitored at 15:29 (B) and 20 minutes later, the amplitude of the tongue's MEP was disappeared at 15:46 (arrow) (A). There were no abnormal findings in other muscles. MEP, motor evoked potential; EMG, electromyography.

the action of poking well on the right side. Therefore, the patient took a videofluoroscopy swallowing study (VFSS) on May 26, 2016 for augmenting the evaluating pattern of the dysphagia. On the VFSS, an incomplete laryngeal closure with decreased laryngeal elevation and epiglottic folding were observed. An incidence of deep penetration was observed on thin liquid and the Functional Oral Intake Scale was noted at a number 5 . Therefore, the patient was recommended a modified diet as nectar-like consistency and underwent swallowing therapy. At subsequent review around 5 weeks later, the tongue deviation to the right side was remained minimally. However, tongue fasciculation and tongue atrophy were not observed at that time. On the follow-up VFSS, the laryngeal closure was improved and penetration was observed on cup drinking, only Functional Oral Intake Scale was 7. Thus, the patient did not need a modified diet and further assistance from pursuing a swallowing therapy at that time.

\section{Case 2}

A median suboccipital craniectomy was made, and the floor of the fourth ventricle was subsequently exposed. The whitish tumor was recognized under the surface of the fourth ventricle, and well demarcated with a capsule. After pulling the capsule to remove the tumor, the repetitive small tonic discharges from tongue muscles were detected (Fig. 4B). After 20 minutes from tonic discharge, the amplitude of MEP from tongue muscles was reduced to the $50 \%$ of its baseline (Fig. 4A). Therefore, the surgeon discontinued invasive capsule removal at that time. Instead, a decompression of the tumor was conducted and an outflow track of cerebrospinal fluid was secured. Although the amplitude of hypoglossal MEP was recovered partly, it was still below $50 \%$ of its baseline at the end of surgery. However, there was no evidence of hypoglossal nerve deficits such as tongue deviation, tongue fasciculation. And the diplopia and dizziness noted in the participant patient were resolved after the surgery.

\section{DISCUSSION}

During infratentorial lesion surgery such as the cerebellum, brainstem, or floor of fourth ventricle, the LCN are placed at high risk of injury [1-3]. It is noted that the deficits of the LCN can result in dysarthria, dysphagia and a decreased quality of life. Thus, IOM of LCN is important 


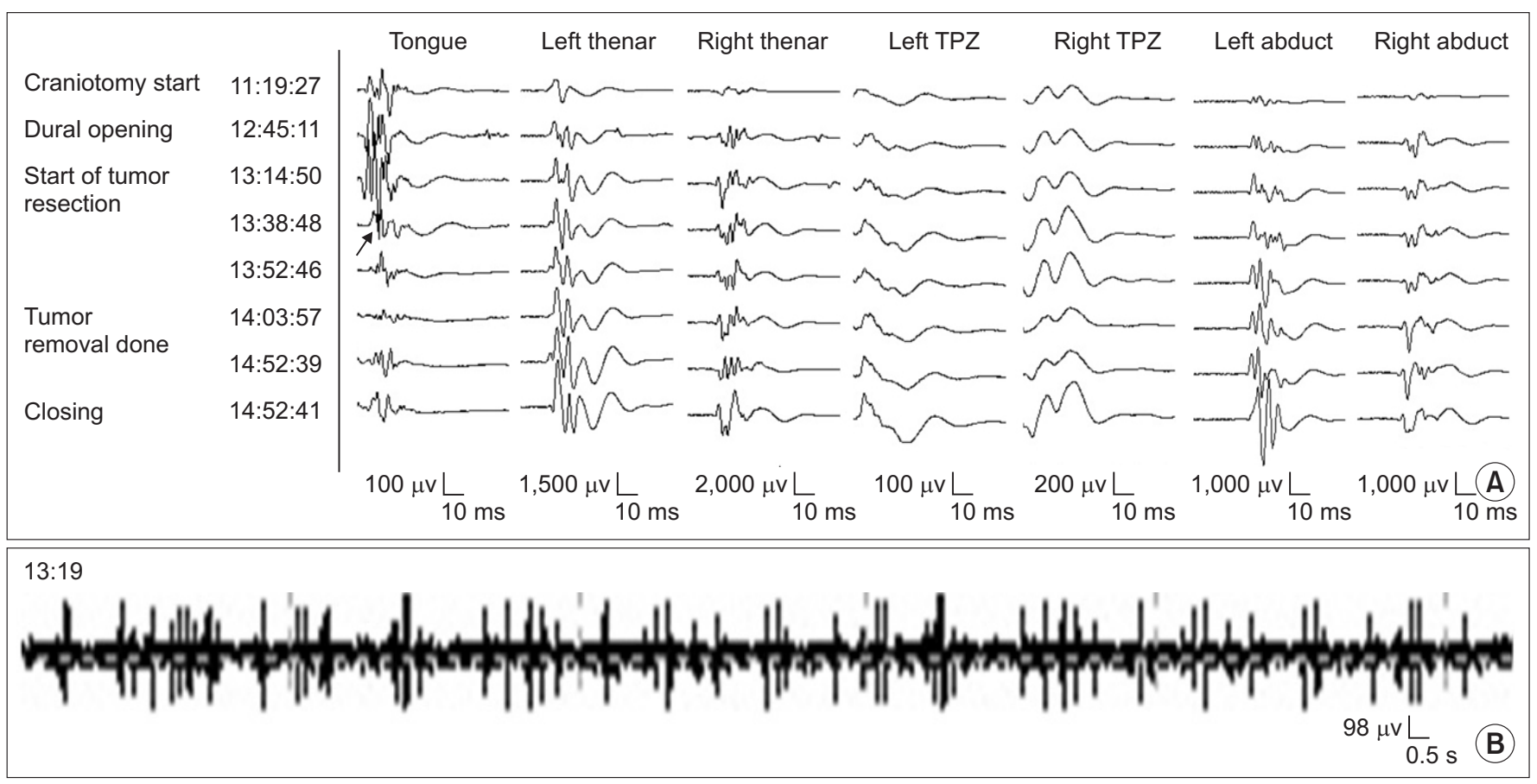

Fig. 4. The tongue and other muscles MEP (A) and spontaneous tongue EMG (B) recorded in case 2. The small tonic discharge was detected from the spontaneous EMG of tongue muscles at 13:19 (B). After 20 minutes, the amplitude of tongue's MEP was reduced at 13:38 to the $50 \%$ of its baseline (arrow) (A). There were no abnormal findings in other muscles. MEP, motor evoked potential; EMG, electromyography.

to utilize to prevent the postoperative complications in infratentorial surgery, because the IOM can give immediate feedback when the nerve is in danger of injury during surgical manipulation [3].

To the best of our knowledge, there has been one study investigating the predictive value of TcMEP from the tongue muscles for a noted postoperative functional outcome [6]. The study reported a non-significant trend between the final-to-baseline MEP ratio of the MEP width and tongue deviation. However, the ratio is considered impractical as an ongoing warning criteria, for use during a surgery. Generally, the disappearance, amplitude reduction, acute threshold elevation, and morphology simplification have been used for characterizing a muscle MEP warning criteria $[6,10]$. In addition, the patients were simply classified by either being present or absent of dysphagia by asking a question, and not evaluated objectively. Therefore, it is still unclear whether hypoglossal MEP can be used to predict postoperative functional deficits such as dysphagia and dysarthria.

For the IOM of cranial nerves, sEMG, tEMG and TcMEP could be utilized in this case. Although many studies have demonstrated that the deterioration of cranial nerves can be monitored using TcMEP [7-10], most of those case studies are restricted in the review of facial nerves $[8,10]$. And the reports about using TcMEP to monitor the deterioration of LCN have been rare and limited in a review of the glossopharyngeal (CN IX) and vagus (CN X) nerves $[7,9,10]$. Fukuda et al. [9] analyzed an associated correlation between the pharyngeal MEP (PhMEP) and postoperative swallowing function in 21 patients. Similarly, Ito et al. [10] demonstrated the usefulness of the TcMEP from the vocalis muscles for detecting the deterioration of the vagus nerve during surgery. However, performing the TcMEP of the CN IX and CN X has some difficulties with the electrode placements $[9,10]$. For the IOM of these nerves, the recording electrodes are inserted in the pharyngeal wall, soft palate, or vocal cord and the appropriate approach to these areas requires complicated and specialized skills to be utilized for optimum outcomes $[9,10]$. Using an endotracheal tube with surface electrodes instead of a needle electrode also can be used for monitoring CN X. Although it should be noted that this method can usually work to detect not the pharyngeal branch of CN X, but the recurrent laryngeal nerve instead. Thus, this device is noted as having restrictions to predict any 
applicable postoperative swallowing complications. Additionally, this device is more expensive than the cost of using a conventional needle electrode.

There are some advantages of using the TcMEP for monitoring the deficits of CN XII, rather than using sEMGs and/or t-EMGs. Conventionally, the hypoglossal nerve was monitored by s-EMGs and t-EMGs [2-4]. But, these methods cannot give continuous real-time monitoring in the early steps of surgery, because the CMAP monitoring needs to have proximity of the nerve in the surgical field, to accomplish that goal [10]. Thus, these methods are unusable if the target nerve is inaccessible in surgical manipulation. In contrast, the TcMEP is an indirect monitoring method in this case, and the closeness of monitoring to the nerve is unnecessary. Furthermore, the intact response of muscle from the EMG does not always mean that there is preservation of the intact quality of the nerve, because the distal part from the nerve damage can produce signals to the target muscles. On the other hand, the TcMEP can provide the information of the functional integrity, from the corticobulbar tract to the hypoglossal nerve [10] and is in the position to better reflect functional deficits. Thus, the TcMEP is recommended to detect the functional deterioration of the hypoglossal nerve.

The present report has some limitations as noted in the study. This is the first case reviewed concerning the usefulness of the amplitude of MEP from CN XII, and before this time, a warning criterion of hypoglossal MEP deficits has not been established in a prior literature review. Therefore, further studies of intraoperative hypoglossal MEP monitoring in larger populations are needed to establish a verifiable reliability and a warning criterion for the hypoglossal MEP monitoring.

In conclusion, the disappearance of the hypoglossal MEP recorded from the tongue could be a predictor of postoperative hypoglossal nerve deficit and subsequent dysphagia. Therefore, future research should explore the development and test more structured monitoring methods in large number of patients.

\section{CONFLICT OF INTEREST}

No potential conflict of interest relevant to this article was reported.

\section{REFERENCES}

1. Jahangiri FR, Minhas M, Jane J. Preventing lower cranial nerve injuries during fourth ventricle tumor resection by utilizing intraoperative neurophysiological monitoring. Neurodiagn J 2012;52:320-32.

2. Schlake HP, Goldbrunner RH, Milewski C, Krauss J, Trautner H, Behr R, et al. Intra-operative electromyographic monitoring of the lower cranial motor nerves (LCN IX-XII) in skull base surgery. Clin Neurol Neurosurg 2001;103:72-82.

3. Topsakal C, Al-Mefty O, Bulsara KR, Williford VS. Intraoperative monitoring of lower cranial nerves in skull base surgery: technical report and review of 123 monitored cases. Neurosurg Rev 2008;31:45-53.

4. Holdefer RN, Kinney GA, Robinson LR, Slimp JC. Alternative sites for intraoperative monitoring of cranial nerves X and XII during intracranial surgeries. J Clin Neurophysiol 2013;30:275-9.

5. Ishikawa M, Kusaka G, Takashima K, Kamochi H, Shinoda S. Intraoperative monitoring during surgery for hypoglossal schwannoma. J Clin Neurosci 2010;17: 1053-6.

6. Kullmann M, Tatagiba M, Liebsch M, Feigl GC. Evaluation of the predictive value of intraoperative changes in motor-evoked potentials of caudal cranial nerves for the postoperative functional outcome. World Neurosurg 2016;95:329-34.

7. Fukuda M, Takao T, Hiraishi T, Yajima N, Saito A, Fujii Y. Novel devices for intraoperative monitoring of glossopharyngeal and vagus nerves during skull base surgery. Surg Neurol Int 2013;4:97.

8. Akagami R, Dong CC, Westerberg BD. Localized transcranial electrical motor evoked potentials for monitoring cranial nerves in cranial base surgery. Neurosurgery 2005;57(1 Suppl):78-85.

9. Fukuda M, Oishi M, Hiraishi T, Saito A, Fujii Y. Pharyngeal motor evoked potentials elicited by transcranial electrical stimulation for intraoperative monitoring during skull base surgery. J Neurosurg 2012;116: 605-10.

10. Ito E, Ichikawa M, Itakura T, Ando H, Matsumoto $\mathrm{Y}$, Oda K, et al. Motor evoked potential monitoring of the vagus nerve with transcranial electrical stimulation during skull base surgeries. J Neurosurg 2013;118:195201. 